TRANSACTIONS OF THE

AMERICAN MATHEMATICAL SOCIETY

Volume 350, Number 1, January 1998, Pages 321-329

S 0002-9947(98)01792-9

\title{
WIDTHS OF SUBGROUPS
}

\author{
RITA GITIK, MAHAN MITRA, ELIYAHU RIPS, AND MICHAH SAGEEV
}

\begin{abstract}
We say that the width of an infinite subgroup $H$ in $G$ is $n$ if there exists a collection of $n$ essentially distinct conjugates of $H$ such that the intersection of any two elements of the collection is infinite and $n$ is maximal possible. We define the width of a finite subgroup to be 0 . We prove that a quasiconvex subgroup of a negatively curved group has finite width. It follows that geometrically finite surfaces in closed hyperbolic 3-manifolds satisfy the $k$-plane property for some $k$.
\end{abstract}

\section{INTRODUCTION}

A subgroup $H$ of a group $G$ is called malnormal in $G$ if for any $g \in G \backslash H$ the intersection $g^{-1} \mathrm{Hg} \cap \mathrm{H}$ is trivial. Malnormality may be thought of as the opposite of normality, and may be geometrically interpreted as an annulus-free condition. Most subgroups are not normal but are also far from being malnormal. For example, the subgroup generated by $a$ in the Baumslag-Solitar group $B(1,2)=\left\langle a, b \mid b a b^{-1}=a^{2}\right\rangle$ is not normal, but any finite collection of its conjugates has infinite intersection. In this paper we generalize the concept of malnormality in two ways.

Definition 0.1. Let $H$ be a subgroup of a group $G$. We say that the elements $\left\{g_{i} \mid 1 \leq i \leq n\right\}$ of $G$ are essentially distinct if $H g_{i} \neq H g_{j}$ for $i \neq j$. Conjugates of $H$ by essentially distinct elements are called essentially distinct conjugates.

Note that we are abusing notation slightly here, as a conjugate of $H$ by an element belonging to the normalizer of $H$ but not belonging to $H$ is still essentially distinct from $H$. Thus in this context a conjugate of $H$ records (implicitly) the conjugating element.

Definition 0.2. We say that the height of an infinite subgroup $H$ in $G$ is $n$ if there exists a collection of $n$ essentially distinct conjugates of $H$ such that the intersection of all the elements of the collection is infinite and $n$ is maximal possible. We define the height of a finite subgroup to be 0 .

Definition 0.3. We say that the width of an infinite subgroup $H$ in $G$ is $n$ if there exists a collection of $n$ essentially distinct conjugates of $H$ such that the intersection of any two elements of the collection is infinite and $n$ is maximal possible. We define the width of a finite subgroup to be 0 .

The width can be thought of as an algebraic version of the $k$-plane property which was introduced by P. Scott in [Scott]. The $k$-plane property says roughly

Received by the editors September 19, 1995 and, in revised form, March 25, 1996.

1991 Mathematics Subject Classification. Primary 20F32, 57M07.

Research of the first author supported in part by NSF grant DMS 9022140.

(C)1998 American Mathematical Society 
that out of any $k$ distinct planes corresponding to lifts of an immersed surface in a 3-manifold to its universal cover at least two are disjoint. This property has important applications in 3-manifold theory. For example, the 4-plane property was used in $[\mathrm{H}-\mathrm{S}]$ to prove that homotopy equivalence implies homeomorphism for certain 3-manifolds.

It is easy to see that infinite malnormal subgroups have height and width 1 , subgroups of finite index have finite height and width, and infinite normal subgroups of infinite index have infinite height and width. The main result of this paper is the following:

The Main Theorem. A quasiconvex subgroup of a negatively curved group has finite width; hence it has finite height.

Corollary. As any quasi-Fuchsian subgroup of the fundamental group of a closed hyperbolic 3-manifold is quasiconvex, all such subgroups have finite width. Hence quasi-Fuchsian subgroups of closed hyperbolic 3-manifolds have the $k$-plane property for some $k$.

We give two different proofs of the Main Theorem which stem from two different perspectives, one viewing negatively curved groups as a generalization of free groups, the other viewing negatively curved groups as a generalization of cocompact Kleinian groups. The first proof, found jointly by the first and the third authors, was motivated by their research about intersections of conjugates in free groups, (cf. $[\mathrm{G}-\mathrm{R}]$ ). It uses basic geometry of negatively curved groups and the pigeonhole principle, and is given in Section 1. The second proof, discovered independently by the second and the fourth authors, originated in the study of immersed incompressible surfaces in 3-manifolds. It makes use of the action of a negatively curved group on its boundary and of the behavior of limit sets under this action. The argument is modeled on the proof that quasi-Fuchsian surface subgroups satisfy the $k$-plane property for some $k$ (cf. [Mi], [R-S]), and is given in Section 2 .

\section{Counting Cosets}

Let $X$ be a set, let $X^{*}=\left\{x, x^{-1} \mid x \in X\right\}$, and for $x \in X$ define $\left(x^{-1}\right)^{-1}=x$. Recall that the Cayley graph of $G=\langle X \mid R\rangle$ denoted by $\Gamma$ is an oriented graph whose set of vertices is $G$ and the set of edges is $G \times X^{*}$, such that an edge $(g, x)$ begins at the vertex $g$ and ends at the vertex $g x$. Since the Cayley graph depends on the generating set of the group, we work with a fixed generating set. The word metric on $\Gamma$, denoted by $\rho$, is defined by assigning to each edge length 1 . A geodesic in $\Gamma$ is a shortest path joining two vertices. A geodesic triangle in $\Gamma$ is a closed path $p=p_{1} p_{2} p_{3}$, where each $p_{i}$ is a geodesic. A group $G$ is $\delta$-negatively curved if any side of any geodesic triangle in $\Gamma$ belongs to the $\delta$-neighborhood of the union of the other two sides. As usual, we assume that all negatively curved groups are finitely generated. A subset $S$ of $G$ is $K$-quasiconvex in $G$ if any geodesic in $\Gamma$ with endpoints in $S$ belongs to the $K$-neighborhood of $S$. We say that $S$ is quasiconvex in $G$ if it is $K$-quasiconvex in $G$ for some $K$. It is well-known that a quasiconvex subgroup of a finitely generated group is finitely generated ([Sh]).

A word in $X$ is any finite sequence of elements of $X^{*}$. Denote the set of all words in $X$ by $W(X)$, and denote the equality of two words by "三". The length of the element $g$ in the group $G$, denoted by $|g|$, is the length of a shortest word in $X$ representing $g$. 
Definition 1.1. The label of a path $p=\left(g_{1}, x_{1}\right)\left(g_{1} x_{1}, x_{2}\right) \cdots\left(g_{1} x_{1} \cdots x_{n-1}, x_{n}\right)$ in $\Gamma$ is the function $\operatorname{Lab}(p) \equiv x_{1} x_{2} \ldots x_{n} \in W(X)$. As usual, we identify the word $\operatorname{Lab}(p)$ with the corresponding element in $G$.

Let $\gamma$ be a geodesic in $\Gamma$ with endpoints at $v$ and $w$. The inverse of $\gamma$ is denoted by $\bar{\gamma}$. The length of $\gamma$, which is equal to $\rho(v, w)$, is denoted by $|\gamma|$.

Lemma 1.2. Let $H$ be a $K$-quasiconvex subgroup of a $\delta$-negatively curved group $G$. If a shortest representative of the double coset $\mathrm{HgH}$ has length greater than $2 \mathrm{~K}+2 \delta$, then the intersection $H \cap g^{-1} H g$ consists of elements shorter than $2 K+8 \delta+2$; hence it is finite.

Proof. As the cardinality of the set $H \cap g^{-1} \mathrm{Hg}$ is invariant under conjugation, we can assume without loss of generality that $g$ is a shortest representative of the coset $g H$. Consider $h \in H \cap g^{-1} H g$. Let $h_{0} \in H$ be such that $h=g^{-1} h_{0} g$. Let $p_{h}, p_{1}, p_{h_{0}}$ and $p_{2}$ be geodesics in $\Gamma$ such that $p_{h}$ begins at 1 and ends at $h, p_{1}$ begins at 1 and ends at $g^{-1}, p_{h_{0}}$ begins at $g^{-1}$ and ends at $g^{-1} h_{0}$ and $p_{2}$ begins at $g^{-1} h_{0}$ and ends at $g^{-1} h_{0} g=h$. Hence $\operatorname{Lab}\left(p_{h}\right)=h, \operatorname{Lab}\left(p_{1}\right)=g^{-1}, \operatorname{Lab}\left(p_{2}\right)=g$ and $\operatorname{Lab}\left(p_{h_{0}}\right)=h_{0}$. Consider the geodesic 4 -gon formed by $p_{1}, p_{h}, p_{2}$ and $p_{h_{0}}$. Let $v$ be a middle vertex of $p_{h}$, so $|\rho(1, v)-\rho(v, h)| \leq 1$. Let $q$ be the initial subpath of $p_{h}$ connecting 1 to $v$. As $H$ is $K$-quasiconvex and $h \in H$, there exists a path $s$ shorter than $K$ which begins at $v$ and ends at an element from $H$. Let $t$ be a shortest path which begins at $v$ and ends at some vertex $w$ of $p_{h_{0}}$, and let $q^{\prime}$ be the initial subpath of $p_{h_{0}}$ connecting $g^{-1}$ to $w$. As $H$ is $K$-quasiconvex and $h_{0} \in H$, there exists a path $s^{\prime}$ shorter than $K$ which begins at an element from $g^{-1} H$ and ends at $w$. Then $g=\operatorname{Lab}\left(q^{\prime} \bar{s}^{\prime}\right) \operatorname{Lab}\left(s^{\prime} \bar{t} s\right) \operatorname{Lab}(\bar{s} \bar{q})$. But $\operatorname{Lab}(q s) \in H$ and $\operatorname{Lab}\left(q^{\prime} \bar{s}^{\prime}\right) \in H$, hence $\operatorname{Lab}\left(s^{\prime} \bar{t} s\right) \in H g H$. As any element in $H g H$ is longer than $2 K+2 \delta$, so is $\operatorname{Lab}\left(s^{\prime} \bar{t} s\right)$. But then $|t|>2 K+2 \delta-|s|-\left|s^{\prime}\right|>2 \delta$; hence $\rho\left(v, p_{h_{0}}\right)>2 \delta$. As $G$ is $\delta$-negatively curved, any side of any geodesic 4 -gon in $\Gamma$ belongs to the $2 \delta$-neighborhood of the union of the other three sides, so $v$ belongs to the $2 \delta$-neighborhood of $p_{1} \cup p_{2}$. Assume that there exists a path $y$ of length less than $2 \delta$ which begins at a vertex $u$ of $p_{1}$ and ends at $v$. Then $p_{1}=p_{1}^{\prime} p_{1}^{\prime \prime}$, where $p_{1}^{\prime}$ ends at $u$. As $g$ is a shortest representative of $g H$, and as $g=\operatorname{Lab}\left(\bar{p}_{1}\right)=\operatorname{Lab}\left(\bar{p}_{1}^{\prime \prime} y s\right) \operatorname{Lab}(\bar{s} \bar{q})$, it follows that $|g|=\left|p_{1}^{\prime}\right|+\left|p_{1}^{\prime \prime}\right| \leq|s|+|y|+\left|p_{1}^{\prime \prime}\right|$. Hence $\left|p_{1}^{\prime}\right| \leq K+2 \delta$. But then $|q| \leq\left|p_{1}^{\prime}\right|+|y| \leq K+4 \delta$, so $|h|<2|q|+2<2 K+8 \delta+2$. Similarly, if $v$ belongs to the $2 \delta$-neighborhood of $p_{2}$, it follows that $|h|<2 K+8 \delta+2$, proving the Lemma.

Lemma 1.3. Let $G$ be a $\delta$-negatively curved group, let $M=42 \delta+12 K$, and let $m$ be the number of elements of $G$ with length not greater than $M$. Let $\left\{g_{i}^{-1} H g_{i}, 1 \leq\right.$ $i \leq n\}$ be a collection of essentially distinct conjugates of a $K$-quasiconvex subgroup $H$ of $G$ such that $g_{i}$ is a shortest representative of the coset $H g_{i}$ and $\left|g_{i}\right| \leq\left|g_{n}\right|$, for $1 \leq i<n$. If for $i \neq n$ all the products $g_{i} g_{n}^{-1}$ belong to the same double coset $H s H$ with $|s| \leq 2 K+2 \delta$, then $n \leq m$.

Proof. As $g_{i} g_{n}^{-1} \in H s H$, for $1 \leq i<n$ and $|s| \leq 2 K+2 \delta$ there exist $h_{i}$ and $k_{i}$ in $H$, and $s_{i} \in H s H$ such that $g_{i} g_{n}^{-1}=h_{i} s_{i} k_{i},\left|s_{i}\right| \leq 3 \delta+2 K$, and $h_{i} s_{i} k_{i}$ is shortest possible. The Lemma is an immediate consequence of the following technical result.

Proposition. The length of $k_{i}$ is less than $\frac{M}{2}$ for $n>i \geq 1$.

Indeed, the Proposition implies that $\left|s_{i} k_{i}\right|<3 \delta+2 K+\frac{M}{2}<M$ for all $1 \leq i<n$. Hence the pigeonhole principle implies that if $n>m$, then for some pair of indices 
$1 \leq i<j<n$ the elements $s_{i} k_{i}$ and $s_{j} k_{j}$ are equal. But then $g_{i} g_{n}^{-1}=h_{i} s_{i} k_{i}$ and $g_{j} g_{n}^{-1}=h_{j} s_{i} k_{i}$. Therefore $H g_{i}=H g_{j}$, contradicting the assumption that the conjugates $g_{i}^{-1} H g_{i}$ and $g_{j}^{-1} H g_{j}$ are essentially distinct.

Proof of the Proposition. To avoid awkward notation we prove the Proposition for $k_{1}$. The proof for other values of the index $i$ is identical. Let $\alpha$ be a geodesic in $\Gamma$ beginning at $g_{1}^{-1}$ and ending at 1 , so $\operatorname{Lab}(\alpha)=g_{1}$, and let $\omega$ be a geodesic in $\Gamma$ beginning at $g_{n}^{-1}$ and ending at 1 , so $\operatorname{Lab}(\omega)=g_{n}$.

Let $\eta, \sigma$ and $\kappa$ be geodesics in $\Gamma$ labeled with $h_{1}, s_{1}$ and $k_{1}$ correspondingly, such that $\eta \sigma \kappa \omega \bar{\alpha}$ is a closed path. In fact, it is a geodesic pentagon. As $G$ is $\delta$-negatively curved, any side of any geodesic pentagon in $\Gamma$ belongs to the $3 \delta$-neighborhood of the union of the other four sides. Denote the $m$-neighborhood of a set $S$ by $N_{m}(S)$ and recall that the initial and the terminal vertices of $\kappa$ are $g_{n}^{-1} k_{1}^{-1}$ and $g_{n}^{-1}$. Let $u$ be the vertex in $\kappa$ such that $u \in N_{3 \delta}(\eta \cup \sigma)$ and $\rho\left(u, g_{n}^{-1}\right)$ is minimal possible, and let $v$ be the vertex in $\kappa$ such that $u \in N_{3 \delta}(\omega)$ and $\rho\left(v, g_{n}^{-1} k_{1}^{-1}\right)$ is minimal possible. Let $\kappa_{1}$ be the initial subpath of $\kappa$ ending at $u$ and let $\kappa_{3}$ be the terminal subpath of $\kappa$ beginning at $v$. If the complement $\kappa_{2}$ of $\kappa_{1} \cup \kappa_{3}$ in $\kappa$ is non-empty, then it is connected and $\kappa_{2} \subset N_{3 \delta}(\alpha)$. In this case $\left|k_{1}\right|=|\kappa|=\left|\kappa_{1}\right|+\left|\kappa_{2}\right|+\left|\kappa_{3}\right|$. If $\kappa_{2}$ is empty, then $\left|k_{1}\right|=|\kappa|<\left|\kappa_{1}\right|+\left|\kappa_{3}\right|$. In either case, the following Claims imply that $\left|k_{1}\right|<(6 \delta+K)+(9 \delta+K)+(6 \delta+4 K)=\frac{M}{2}$, proving the proposition.

Claim 1. $\left|\kappa_{3}\right| \leq 6 \delta+K$.

Indeed, let $y^{\prime}$ be a path shorter than $3 \delta$ which begins at $v$ and ends at a vertex $v^{\prime}$ of $\omega$. Let $\omega^{\prime} \omega^{\prime \prime}$ be a decomposition of $\omega$ such that $v^{\prime}$ is the terminal vertex of $\omega^{\prime}$. As $H$ is $K$-quasiconvex and as $L a b(\kappa) \in H$, there exists a path $y^{\prime \prime}$ shorter than $K$ which ends at $v$ such that $\operatorname{Lab}\left(y^{\prime \prime} \kappa_{3}\right) \in H$. As $g_{n}$ is a shortest representative of $H g_{n}$, it follows that $\left|g_{n}\right|=|\omega|=\left|\omega^{\prime}\right|+\left|\omega^{\prime \prime}\right| \leq\left|y^{\prime \prime} y^{\prime} \omega^{\prime \prime}\right| \leq K+3 \delta+\left|\omega^{\prime \prime}\right|$; hence $\left|\omega^{\prime}\right| \leq 3 \delta+K$. But then $\left|\kappa_{3}\right| \leq\left|\omega^{\prime}\right|+\left|y^{\prime}\right| \leq 6 \delta+K$.

Claim 2. $\left|\kappa_{2}\right| \leq 9 \delta+K$.

Let $\alpha^{\prime} \alpha^{\prime \prime}$ be the decomposition of $\alpha$ such that no point of $\alpha^{\prime \prime}$ belongs to $N_{3 \delta}(\kappa)$ and $\alpha^{\prime \prime}$ is the maximal possible terminal subpath of $\alpha$. Then $\kappa_{2} \subset N_{3 \delta}\left(\alpha^{\prime}\right)$; hence $\left|\kappa_{2}\right| \leq\left|\alpha^{\prime}\right|+6 \delta$. It remains to show that $\left|\alpha^{\prime}\right|<3 \delta+K$. Indeed, let $a$ be the terminal vertex of $\alpha^{\prime}$. Then there exists a path $f^{\prime}$ of length less than $3 \delta$ which begins at a vertex $a^{\prime}$ in $\kappa$ and ends at $a$. As $H$ is $K$-quasiconvex and as $L a b(\kappa) \in H$, there exists a path $f^{\prime \prime}$ of length less than $K$, which begins at $g_{n}^{-1} H$ and ends at $a^{\prime}$. As $g_{n}$ is the shortest representative of $H g_{n}$, it follows that $|\omega| \leq\left|\alpha^{\prime \prime}\right|+\left|f^{\prime}\right|+\left|f^{\prime \prime}\right| \leq\left|\alpha^{\prime \prime}\right|+3 \delta+K$. But $|\omega| \geq|\alpha|=\left|\alpha^{\prime}\right|+\left|\alpha^{\prime \prime}\right|$; hence $\left|\alpha^{\prime}\right| \leq 3 \delta+K$.

Claim 3. $\left|\kappa_{1}\right| \leq 6 \delta+4 K$.

If $u \in N_{3 \delta}(\eta)$, let $b$ be a path of length less than $3 \delta$ beginning at $u$ and ending at a vertex $u^{\prime}$ of $\eta$. Let $c$ and $d$ be paths of length less than $K$ such that $c$ begins at $g_{n}^{-1} H$ and ends at $u$, and $d$ begins at cosets $u^{\prime}$ and ends at $g_{1}^{-1} H$. Then $L a b(c b d) \in H s H$ and $|c b d| \leq 2 K+3 \delta$. Let $\eta^{\prime}$ be the initial subpath of $\eta$ terminating at $u^{\prime}$. As $\operatorname{Lab}\left(\eta^{\prime} d\right) \in H, \operatorname{Lab}\left(c \kappa_{1}\right) \in H$; and as $h_{1} s_{1} k_{1}$ is shortest possible, it follows that $|\eta \sigma \kappa| \leq\left|\eta^{\prime} d\right|+|c b d|+\left|c \kappa_{2} \kappa_{3}\right|$, which implies that $|\eta|+|\sigma|+|\kappa|-\left|\eta^{\prime}\right|-\left|\kappa_{2} \kappa_{3}\right| \leq$ $2|c|+2|d|+|b| \leq 3 \delta+4 K$. As $|\eta| \geq\left|\eta^{\prime}\right|$ and as $|\kappa|=\left|\kappa_{1}\right|+\left|\kappa_{2}\right|+\left|\kappa_{3}\right|$, it follows that $\left|\kappa_{1}\right| \leq 3 \delta+4 K$.

If $u \in N_{3 \delta}(\sigma)$, note that $|\sigma|=\left|s_{1}\right|<3 \delta+2 K$. Hence in this case, $\left|\kappa_{1}\right| \leq$ $|\sigma|+3 \delta \leq 6 \delta+2 K$. 
Proof of the Main Theorem. Let $H$ be a $K$-quasiconvex subgroup of a $\delta$-negatively curved group $G$, and let $m$ be as in Lemma 1.3. As $G$ is finitely generated, there exists a finite number, say $N$, of elements in $G$ of length not greater than $2 K+2 \delta$. We claim that $H$ has width less than $m N$. Indeed, let $\left\{g_{i}^{-1} H g_{i} \mid 1 \leq i \leq n\right\}$ be a collection of essentially distinct conjugates of $H$, such that $g_{i}$ is a shortest representative of the coset $H g_{i}$ and $\left|g_{i}\right| \leq\left|g_{n}\right|$, for $1 \leq i<n$. Lemma 1.3 implies that if $n \geq m N$ there exists an index $i<n$ such that the length of a shortest representative of the double coset $H_{i} g_{n}^{-1} H$ is greater than $2 K+2 \delta$. But then Lemma 1.2 implies that the intersection $g_{i}^{-1} H_{i} \cap g_{n}^{-1} H g_{n}$ is finite, proving the Main Theorem.

\section{Limit Sets}

We recall some generalities about negatively curved groups. For a thorough discussion of these matters see, for instance, [Gr 1], [C-D-P] or [G-H]. The Gromov boundary of the Cayley graph $\Gamma$, denoted by $\partial \Gamma$, is the collection of equivalence classes of geodesic rays $r:[0, \infty) \rightarrow \Gamma$ with $r(0)=1$, where rays $r_{1}$ and $r_{2}$ are equivalent if $\sup \left\{\rho\left(r_{1}(t), r_{2}(t)\right)\right\}<\infty$. The Gromov inner product of elements $a$ and $b$ in $\Gamma$ is defined by $(a, b)=\frac{1}{2}\{\rho(a, 1)+\rho(b, 1)-\rho(a, b)\}$. It extends naturally to an inner product on $\partial \Gamma$, defining a metric on $\partial \Gamma$ (cf. $[\mathrm{G}-\mathrm{H}])$. This metric, denoted by $d$, is called the visual metric on $\partial \Gamma$. The diameter of a set in the visual metric will be called its visual diameter. The limit set $\Lambda(S)$ of a subset $S$ of $\Gamma$ is defined in the usual way as the collection of accumulation points of $S$ in $\partial \Gamma$. It is easy to see that if $S$ is a $K$-quasiconvex subset of $\Gamma$, then infinite geodesics that start in $S$ and terminate on the limit set of $S$ lie in a $K$-neighborhood of $S$. Let $H$ be a $K$-quasiconvex subgroup of $G$. As $G$ acts on itself on the left by isometries, it follows that every left coset $g H$ is $K$-quasiconvex. The conjugate $g H^{-1}$ stabilizes the coset $g H$, and every element of $g H^{-1}$ is within distance $|g|$ of an element in $g H$. It follows that $\Lambda(g H)=\Lambda\left(g H g^{-1}\right)$.

Remark 2.1. Since cosets are disjoint, any ball of finite radius in $\Gamma$ has non-empty intersection with only finitely many left cosets of $H$.

This observation has the following immediate corollary:

Theorem 2.2. A quasiconvex subgroup of a negatively curved group has finite height.

Proof. Let $H$ be a $K$-quasiconvex subgroup of a negatively curved group $G$, and let $\left\{g_{1}^{-1} H g_{1}, \ldots, g_{m}^{-1} H g_{m}\right\}$ be a set of essentially distinct conjugates of $H$ such that $\bigcap g_{i}^{-1} H g_{i}$ is infinite. Then $\bigcap g_{i}^{-1} H g_{i}$ contains an element of infinite order which gives rise to a geodesic $l$ with endpoints in $\bigcap \Lambda\left(g_{i}^{-1} H g_{i}\right)=\bigcap \Lambda\left(g_{i}^{-1} H\right)$. As any vertex, say $v$, quasiconvex in $l$ lies in a $K$-neighborhood of the corresponding left $g_{i}^{-1} H$, all the corresponding left cosets intersect the ball of radius $K$ about $v$. Remark 2.1 implies that there exist a finite number $n$ of left cosets which intersect this ball; thus $m \leq n$, so the height of $H$ is at most $n$.

Thus the asymptotics of $H$ and its conjugates establishes finite height. To prove finite width, one needs a better understanding of the asymptotics. We will be interested in intersection patterns of limit sets of conjugates, so that the following definitions will be useful. 
Definition 2.3. Let $\mathcal{L}$ denote the collection of all the translates of $\Lambda(H)$ under the action of $G$. A subset $S \subset \mathcal{L}$ is said to have non-trivial intersection if the intersection of all elements of $S$ is non-empty; $S$ is said to be full if every pair of elements of $S$ intersect.

We start with the following well-known lemma (cf. [G-H]) which says that quasiconvex sets lying far away from the origin are small in the visual metric.

Lemma 2.4. For every $R>0$ there exists $\epsilon(R)$ such that every $K$-quasiconvex subset of $G$ which does not intersect the ball of radius $R$ around 1 has visual diameter less than $\epsilon(R)$, and $\epsilon(R) \rightarrow 0$ as $R \rightarrow \infty$.

This allows us to estimate the number of conjugates whose diameter is bounded from below.

Corollary 2.5. For given $\epsilon>0$ the set $\{\Lambda \in \mathcal{L}$ : visual diameter of $\Lambda \geq \epsilon\}$ is finite.

Proof. By Lemma 2.4 there exists some $R$ (depending on $\epsilon$ ) such that for every $\Lambda$ with visual diameter larger than $\epsilon$, any coset whose limit set is $\Lambda$ enters the ball of radius $R$ about the origin. Since there are only finitely many such cosets, the set of such $\Lambda$ is finite.

The following two lemmas tell us how quasi-convex subgroups intersect.

Lemma 2.6. If $H_{1}, \ldots, H_{n}$ is a finite collection of quasiconvex subgroups of $G$, then $\bigcap \Lambda\left(H_{k}\right)=\Lambda\left(\bigcap H_{k}\right)$.

Proof. If $G$ is a cocompact lattice in $\mathbb{H}^{n}$, this result was proved by Swarup and Susskind [Su-Sw] (see also [Gr 2], p.164). We prove the lemma for two $K$-quasiconvex subgroups $A$ and $B$; the argument for general $n$ is analogous. The idea is that all points in $\partial \Gamma$ are conical. Given a point $p \in \Lambda(A) \cap \Lambda(B)$, choose a geodesic beginning at 1 and terminating at $p$. This geodesic lies in a $K$-neighborhood of $A$ and of $B$; hence there exist two sequences of points $\left\{a_{i}\right\} \subset A$ and $\left\{b_{i}\right\} \subset B$, converging to $p$, such that $\rho\left(a_{i}, b_{i}\right)<2 K$. Thus $a_{i}$ and $b_{i}$ differ by some path (word) of length $\leq 2 K$. Since there are only finitely many such words, we may pass to subsequences of $\left\{a_{i}\right\}$ and $\left\{b_{i}\right\}$ such that $a_{i}=b_{i} g$ for some fixed $g$ of length less than $2 K$. Let $c_{i}=a_{i} a_{1}^{-1}=\left(b_{i} g\right)\left(g^{-1} b_{1}^{-1}\right)=b_{i} b_{1}^{-1}$. Then $\left\{c_{i}\right\}$ is a sequence in $A \cap B$ converging to $p$.

The following fact is well-known (cf. [Sh]).

Lemma 2.7. If $H_{1}, \ldots, H_{n}$ is a finite collection of quasiconvex subgroups of $G$, then $\cap H_{k}$ is quasiconvex

Proof. We proceed by induction, so it suffices to prove the theorem for the case $n=2$. Suppose $A$ and $B$ are $K$-quasiconvex subgroups and $A \cap B$ is not quasiconvex. It follows that there exists a sequence of points $\left\{x_{i}\right\}$ in $A \cap B$ such that some geodesic path $l_{i}$ from 1 to $x_{i}$ does not lie in the $r_{i}$-neighborhood of $A \cap B$, where $r_{i} \rightarrow \infty$. Thus we can find points $y_{i}$ along $l_{i}$ such that $B_{r_{i}}\left(y_{i}\right)$, the ball of radius $r_{i}$ about $y_{i}$, does not contain any elements of $A \cap B$. As $A$ and $B$ are $K$-quasiconvex, there exist elements $a_{i} \in A$ and $b_{i} \in B$ within $K$ of $y_{i}$; hence $a_{i}$ and $b_{i}$ differ by an element of $B_{2 K}(1)$. Since $B_{2 K}(1)$ is a finite set, we may pass to a subsequence of $\left\{y_{i}\right\}$ so that $a_{i}=b_{i} g$, where $g \in B_{2 K}(1)$. Now choose $r_{i}>K+\left|a_{1}\right|$. 
Then $a_{i} a_{1}^{-1}=\left(b_{i} g\right)\left(g^{-1} b_{1}^{-1}\right)=b_{i} b_{1}^{-1}$ is an element of $A \cap B$ within $K+\left|a_{1}\right|$ of $y_{i}$, a contradiction.

Corollary 2.8. There exists a bound on the size of a subset of $\mathcal{L}$ with non-trivial intersection.

Proof. By Theorem $2.1 H$ has height $n$ for some $n$. Now suppose $S=\left\{\Lambda\left(H_{1}\right), \ldots\right.$, $\left.\Lambda\left(H_{m}\right)\right\}$ is a subset of $\mathcal{L}$ with non-trivial intersection. Then by Lemma 2.6, this intersection is the limit set of $\bigcap H_{i}$. Thus $\bigcap H_{i}$ is an infinite group, and hence $m \leq n$.

The following lemma ensures that the limit set of $H$ is pushed off itself by most group elements not in $H$. This lemma was independently proven by I. Kapovich and H. Short in [K-S] and by E. Swenson in [Swe].

Lemma 2.9. Let $H$ be a $K$-subgroup of a negatively curved group $G$. If $H<L<G$ and $L$ has the same limit set as $H$, then $[L: H]$ is finite.

Proof. Assume that there exist infinitely many distinct right cosets $\left\{H l_{i}\right\}$ of $H$ in $L$, where $l_{i}$ is a shortest representative of $H l_{i}$ and $\rho\left(l_{i}, 1\right) \geq \rho\left(l_{j}, 1\right)$ for $i>j$. Then $\rho\left(l_{i}, 1\right) \rightarrow \infty$, so after passing to a subsequence if necessary, we have that $l_{i} \rightarrow z \in \partial \Gamma$. Then $z \in \Lambda(L)$, but the Claim following Lemma 2.9 states that $z \notin \Lambda(H)$, contradicting the hypothesis that $\Lambda(H)=\Lambda(L)$. Therefore $H$ has finite index in $L$.

Claim. $z$ does not lie in $\Lambda(H)$.

Proof of the Claim. It is enough to show that for any $h \in H$, the Gromov inner product $\left(h, l_{i}\right)$ is bounded by a constant depending only on $K$ and $\delta$, as this shows that the Gromov inner product $(c, z)$ is bounded by the same constant for any point $c$ in the limit set of $H$. Consider geodesic triangles $T_{i}$ with vertices $h, 1$, and $l_{i}$. Let $[x, y]$ denote the edge of the triangle with vertices $x, y . G$ is $\delta$-negatively curved, so that all triangles are $\delta$-thin. Let $t_{i}, u_{i}$ and $v_{i}$ denote the vertices of the inscribed triangle of $T_{i}$ : that is, $t_{i}, u_{i}$ and $v_{i}$ are vertices on the geodesics $\left[1, l_{i}\right],\left[l_{i}, h\right]$ and $[1, h]$ respectively, such that $\rho\left(1, t_{i}\right)=\rho\left(1, v_{i}\right), \rho\left(l_{i}, t_{i}\right)=\rho\left(l_{i}, u_{i}\right)$ and $\rho\left(h, u_{i}\right)=\rho\left(h, v_{i}\right)$. As $v_{i}$ 's lie in a $K$-neighbourhood of $H$, there exist $w_{i} \in H$ such that $\rho\left(v_{i}, w_{i}\right) \leq K$. Also, since triangles are $\delta$-thin, the diameter of the inscribed triangle is at most $2 \delta$ (cf. [Gr 1]). Therefore, $\rho\left(l_{i}, 1\right) \leq \rho\left(l_{i}, w_{i}\right) \leq \rho\left(l_{i}, t_{i}\right)+\rho\left(t_{i}, v_{i}\right)+\rho\left(v_{i}, w_{i}\right)=$ $\rho\left(l_{i}, t_{i}\right)+2 \delta+K$. Hence $\left(h, l_{i}\right)=\rho\left(1, t_{i}\right)=\rho\left(1, l_{i}\right)-\rho\left(l_{i}, t_{i}\right) \leq 2 \delta+K$, proving the claim.

Recall that a quasiconvex subgroup $H$ is said to be maximal in its limit set if $H=\operatorname{stab}(\Lambda(H))$. Lemma 2.9 shows that any quasiconvex subgroup is a finite index subgroup of a quasiconvex subgroup which is maximal in its limit set. We will also need the following.

Lemma 2.10. Let $H$ be a quasi-convex subgroup of $G$ which is maximal in its limit set. Then for any $g \in G \backslash H, g \Lambda(H) \not \subset \Lambda(H)$.

Proof. Assume that $g(\Lambda(H)) \subset \Lambda(H)$. If $g$ has finite order $n$, then $g^{-1}(\Lambda(H))=$ $g^{n-1}(\Lambda(H)) \subset \Lambda(H)$; thus $g(\Lambda(H))=\Lambda(H)$. If $g$ has infinite order, it fixes two distinct points $p$ and $q$, which must lie in the limit set of $g^{n} H$ for every $n$. The geodesic $\lambda$ joining $p$ and $q$ lies in a $K$-neighbourhood of the cosets $g^{n} H$. Let $v$ be a vertex on $\lambda$. Then $g^{n} H$ passes through a $K$-neighbourhood of $v$ for all $n$. 
Thus from Remark 2.1 and the pigeonhole principle $g^{n} \in H$ for some $n$. Hence $\Lambda(H)=g^{n}(\Lambda(H)) \subset g(\Lambda(H))$, so $g(\Lambda(H))=\Lambda(H)$, as before. In any case, the maximality of $H$ in its limit set implies $g \in H$.

Remark 2.11. Let $\Lambda_{1}, \ldots, \Lambda_{l}$ be a collection of limit sets such that $\Lambda_{1} \cap \ldots \cap \Lambda_{l} \neq \emptyset$. By acting on $\Gamma$ by an appropriate group element, we can assume that some geodesic joining a pair of distinct points in $\Lambda_{1} \cap \ldots \cap \Lambda_{l}$ passes through the identity. Then Lemma 2.4 implies that there exists a lower bound $\epsilon_{0}$ (depending only on $K$ ) on the visual diameter of $\Lambda_{1} \cap \ldots \cap \Lambda_{l}$.

Let $L$ be the stabilizer of $\Lambda_{1} \cap \ldots \cap \Lambda_{l}$. Thus $\Lambda(L)=\Lambda_{1} \cap \ldots \cap \Lambda_{l}$.

Lemma 2.12. Given any $\Lambda \in \mathcal{L}$ and a collection $\Lambda_{1}, \ldots, \Lambda_{l}$ such that

1) $\Lambda \cap \Lambda_{i} \neq \emptyset$ for $i=1, \ldots, l$,

2) visual diameter of $\Lambda_{1} \cap \ldots \cap \Lambda_{l} \geq \epsilon_{0}$,

3) $\Lambda_{1} \cap \ldots \cap \Lambda_{l} \cap \Lambda=\emptyset$,

there exist $\epsilon>0$ depending on $L$ and an element $h$ of $L$, depending on $\Lambda$, such that the visual diameter of the translate of $\Lambda$ by $h$ is greater than or equal to $\epsilon$.

Proof. Suppose there exists a sequence $\Lambda_{l+1}, \Lambda_{l+2}, \ldots$ satisfying the conditions of the Lemma, such that the visual diameters of all translates of $\Lambda_{l+i}$ by elements of $L$ are less than $1 / i$. Let $K_{l+i}$ denote the coset of $H$ with limit set $\Lambda_{l+i}$. Let $p_{i} \in L$ and $q_{i} \in K_{l+i}$ be such that $\rho\left(p_{i}, q_{i}\right)=\rho\left(L, K_{l+i}\right)$. Translating each $K_{l+i}$ by an appropriate element of $L$, we can assume that $p_{i}$ is the identity 1 for all $i$. As the $K_{l+i}$ 's are uniformly quasiconvex, being cosets of $H$, Remark 2.1 implies that $\rho\left(p_{i}, q_{i}\right)=\rho\left(1, K_{l+i}\right)=\rho\left(L, K_{l+i}\right) \rightarrow \infty$. Therefore, by Corollary 2.5, $\left\{\Lambda_{l+i}\right\}$ converges (after passage to a subsequence if necessary) to a point $z$. As in the proof of the Claim following Lemma 2.9, one sees that $z$ lies in $\partial \Gamma \backslash \Lambda_{1} \cap \ldots \cap \Lambda_{l}$. But $\Lambda_{l+i} \cap \Lambda_{j} \neq \emptyset$ for $j=1, \ldots, l$. This implies that $z \in \Lambda_{1} \cap \ldots \cap \Lambda_{l}$, a contradiction.

We are now ready to prove the main theorem.

Theorem. H has finite width.

Proof. This argument is similar to the ones in [R-S] and in [Mi] showing that geometrically finite immersed incompressible surfaces satisfy the $k$-plane property. Since it suffices to prove the theorem up to subgroups of finite index, Lemma 2.9 allows us to assume that $H$ is maximal in its limit set. Suppose $H$ does not have finite width. Then for all $n$ we have a collection of full sets $S_{n} \subset \mathcal{L}$ such that the cardinality of $S_{n} \geq n$. Each $S_{n}$ contains a maximal subset $T_{n}$ with non-trivial intersection. As in Remark 2.11, we can translate each $S_{n}$ so that every element of $T_{n}$ has visual diameter larger than $\epsilon_{0}$. Since by Corollary 2.5 the set of translates with visual diameter larger than $\epsilon_{0}$ is finite, we can pass to a subsequence of $\left\{S_{n}\right\}$ so that $T_{n}$ is the same set for each $n$; that is, there exists a set $T \subset S_{n}$ which is a maximal subset with non-trivial intersection in each $S_{n}$. For each $n$ choose an element $\Lambda_{n} \in S_{n} \backslash T$. Let $L$ denote the intersection of the stabilizers of elements in $T$. Then by Lemma 2.12 there exist an $\epsilon_{1}>0$ and an element $g_{n} \in L$ such that translating $S_{n}$ by $g_{n}$ carries $\Lambda_{n}$ to a set of visual diameter greater than $\epsilon_{1}$. Since the set $\left\{\Lambda \in \mathcal{L}\right.$ : visual diameter of $\left.\Lambda \geq \epsilon_{1}\right\}$ is finite, there exists $\Lambda_{0} \in \mathcal{L}$ such that (after passing to a subsequence of $\left\{S_{n}\right\}$ if necessary) $T \cup\left\{\Lambda_{0}\right\} \subset S_{n}$ for all $n$. Let $T^{\prime}=T \cup\left\{\Lambda_{0}\right\}$. Note that $T^{\prime}$ has trivial intersection, as $T$ was a maximal subset with non-trivial intersection. As the cardinality of $S_{n} \rightarrow \infty$, there exists an 
infinite sequence $\left\{\Lambda_{n}\right\}$ with $\Lambda_{n} \in S_{n}$. By Lemmas 2.4 and 2.5 , the visual diameter of $\Lambda_{n}$ approaches 0 , and hence we may pass to a subsequence of $\Lambda_{n}$ converging to some point $z \in \partial \Gamma$. Since $\Lambda_{n}$ intersects every element of $T^{\prime}, z$ is contained in every element of $T^{\prime}$. Hence, we have that $T^{\prime}$ intersects non-trivially, a contradiction.

\section{ACKNOWLEDGEMENTS}

The authors would like to thank Professors A. Casson, H. Rubinstein and G. A. Swarup for their interest in this work and for helpful discussions.

\section{REFERENCES}

[C-D-P] M. Coornaert, T. Delzant and A. Papadopoulos, Géométrie et théorie des groupes, Lecture Notes in Math., vol.1441, Springer Verlag, 1990. MR 92f:57003

[G-H] E. Ghys and P. de la Harpe (editors), Sur les groupes hyperboliques d'après Mikhael Gromov, Progress in Math. vol 83, Birkhauser, Boston, Ma., 1990. MR 92f:53050

[G-R] R. Gitik and E. Rips, Heights of Subgroups, MSRI Preprint 027-95.

[Gr 1] M. Gromov, Hyperbolic Groups, Essays in Group Theory, MSRI series vol.8 (S. M. Gersten, ed.), Springer-Verlag, 1987, pp. 75-263. MR 89e:20070

[Gr 2] Asymptotic Invariants of Infinite Groups, Geometric Group Theory, vol.2; LMS Lecture Notes 182, Cambridge University Press, 1993. MR 95m:20041

[H-S] J. Hass and P. Scott, Homotopy Equivalence and Homeomorphism of 3-Manifolds, Topology 31 (1992), 493-517. MR 94g:57021

[K-S] I. Kapovich and H. Short, Some Remarks on Quasiconvexity, preprint.

[Mi] M. Mitra, Immersed Incompressible Surfaces in Hyperbolic 3-Manifolds, in preparation.

[R-S] H. Rubinstein and M. Sageev, Intersection Patterns of Immersed Incompressible Surfaces, in preparation.

[Scott] P. Scott, There Are No Fake Seifert Fibre Spaces with Infinite $\pi_{1}$, Annals of Math 117 (1983), 35-70. MR 84c:57008

[Sh] H. B. Short, Quasiconvexity and a Theorem of Howson's, Group Theory from a Geometric Viewpoint, Proc. ICTP. Trieste, World Scientific, Singapore, 1991, pp. 168-176. MR 93d:20071

[Su-Sw] P. Susskind and G. A. Swarup, Limit Sets of Geometrically Finite Hyperbolic Groups, Amer. J. Math 114 (1992), 233-250. MR 94d:57066

[Swe] E. Swenson, Limit Sets in the Boundary of Negatively Curved Groups, preprint.

Department of Mathematics, University of Michigan, Ann Arbor, Michigan 48109

E-mail address: ritagtk@math.lsa.umich.edu

Department of Mathematics, University of California, Berkeley, California 94720

E-mail address: mitra@math. berkeley.edu

Institute of Mathematics, Hebrew University, Givat Ram, Jerusalem, 91904, Israel

E-mail address: rips@sunset.huji.ac.il

Department of Mathematics, University of Southampton, Southampton, England

E-mail address: mes@maths.soton.ac.uk 